\title{
Vibrio natriegens as Host for Expression of Multisubunit Membrane Protein Complexes
}

\author{
Lena Schleicher ${ }^{1}$, Valentin Muras ${ }^{1}$, Björn Claussen ${ }^{1}$, Jens Pfannstiel'2, \\ Bastian Blombach ${ }^{3}$, Pavel Dibrov', Günter Fritz ${ }^{1,5}$ and Julia Steuber ${ }^{1 *}$ \\ ${ }^{1}$ Institute of Microbiology, University of Hohenheim, Stuttgart, Germany, ${ }^{2}$ Mass Spectrometry Core Facility, University \\ of Hohenheim, Stuttgart, Germany, ${ }^{3}$ Institute of Biochemical Engineering, University of Stuttgart, Stuttgart, Germany, \\ ${ }^{4}$ Department of Microbiology, University of Manitoba, Winnipeg, MB, Canada, ${ }^{5}$ Institute for Neuropathology, University \\ of Freiburg, Freiburg im Breisgau, Germany
}

Escherichia coli is a convenient host for the expression of proteins, but the heterologous production of large membrane protein complexes often is hampered by the lack of
OPEN ACCESS

Edited by: Kathleen Scott, University of South Florida,

United States

Reviewed by: Jyl S. Matson, University of Toledo, United States Alfonso Soler-Bistue, Instituto de Investigaciones Biotecnológicas (IIB-INTECH), Argentina

*Correspondence: Julia Steuber

julia.steuber@uni-hohenheim.de

Specialty section:

This article was submitted to Microbial Physiology and Metabolism,

a section of the journal

Frontiers in Microbiology

Received: 16 July 2018 Accepted: 04 October 2018 Published: 25 October 2018

Citation:

Schleicher L, Muras V. Claussen B, Pfannstiel J, Blombach B, Dibrov P, Fritz G and Steuber J (2018) Vibrio natriegens as Host for Expression of Multisubunit

Membrane Protein Complexes.

Front. Microbiol. 9:2537. doi: 10.3389/fmicb.2018.02537 specific accessory genes required for membrane insertion or cofactor assembly. In this study we introduce the non-pathogenic and fast-growing Vibrio natriegens as a suitable expression host for membrane-bound proteins from Vibrio cholerae. We achieved production of the primary $\mathrm{Na}^{+}$pump, the $\mathrm{NADH}$ :quinone oxidoreductase (NQR), from $V$. cholerae in an active state, as indicated by increased overall $\mathrm{NADH}$ :quinone oxidoreduction activity of membranes from the transformed $V$. natriegens, and the sensitivity toward $\mathrm{Ag}^{+}$, a specific inhibitor of the NQR. Complete assembly of $\mathrm{V}$. cholerae NQR expressed in $V$. natriegens was demonstrated by BN PAGE followed by activity staining. The secondary transport system Mrp from $V$. cholerae, another membranebound multisubunit complex, was also produced in $V$. natriegens in a functional state, as demonstrated by in vivo $\mathrm{Li}^{+}$transport. $V$. natriegens is a promising expression host for the production of membrane protein complexes from Gram-negative pathogens.

Keywords: Vibrio natriegens, membrane proteins, multisubunit complexes, expression, $\mathrm{Na}^{+}$-translocating NADH:quinone oxidoreductase, multiple resistance and $\mathrm{pH}$ related antiporter, Strep-Tag

\section{INTRODUCTION}

The heterologous expression and purification of proteins allows their biochemical characterization, use in industrial processes, and development of commercial goods (Rosano and Ceccarelli, 2014). The traditional microbial system for heterologous expression of proteins is Escherichia coli with high levels of gene expression and scalability of experiments. However, the production of recombinant proteins, especially membrane-bound proteins, represents the bottleneck of many biotechnological processes. Here we introduce Vibrio natriegens as a new host for heterologous expression of multisubunit membrane proteins in their active state.

Abbreviations: $\mathrm{NQR}, \mathrm{Na}^{+}$-translocating $\mathrm{NADH}$ :quinone oxidoreductase; Mrp, multiple resistance and $\mathrm{pH}$ related antiporter. 
Vibrio natriegens is a Gram-negative, marine, halophilic, nonpathogenic bacterium from the family Vibrionaceae. Although it was first described more than 50 years ago (Payne, 1958), only a few studies of $V$. natriegens exist so far. $V$. natriegens has the fastest growth rate of any known non-pathogenic organism with a doubling time of $<10 \mathrm{~min}$ at optimal growth conditions (Payne, 1958; Eagon, 1962; Weinstock et al., 2016; Hoffart et al., 2017); in comparison, E. coli has a doubling time of $20 \mathrm{~min}$. For this enormous growth rate an extremely high rate of protein synthesis is required, which is due to increased amounts of ribosomes (Aiyar et al., 2002). In addition, genetic engineering tools were successfully developed for the manipulation of cellular metabolism and the heterologous expression of proteins in $V$. natriegens (Weinstock et al., 2016; Hoffart et al., 2017). Recent studies reported a $V$. natriegens-based cell-free protein synthesis system (CFPS) for synthesis of the model protein eGFP (Failmezger et al., 2018). V. natriegens fulfills basic requirements for biotechnological applications (Hoffart et al., 2017): bioreactor cultivations of $V$. natriegens in minimal medium with glucose resulted in a remarkable high qs (specific consumption rate), which was at least two times higher than those of E. coli, Bacillus subtilis, Corynebacterium glutamicum and yeast, the traditional microbial systems. Consequently, $V$. natriegens is a very interesting organism, which could become a tool for different fields of biotechnological research and development.

The heterologous expression of membrane protein complexes in their active state is especially difficult since subunits must integrate into the host's membrane, and must assemble in a correct manner. Both processes have to be coordinated to obtain a fully functional membrane protein complex. Here we examine the potential of $V$. natriegens as a new expression host for membrane proteins from Vibrio cholerae, namely the primary transport system NQR and the secondary transport system Mrp. The NQR is a respiratory enzyme which is widespread among pathogenic bacteria (Steuber et al., 2014a,b). It is composed of six subunits (NqrABCDEF) and contains several cofactors: one FAD, two iron-sulfur centers, one riboflavin, and two covalently bound flavin mononucleotides (FMN). Subunit NqrF harbors FAD and catalyzes the initial oxidation of $\mathrm{NADH}$ which can be specifically inhibited by silver ions (Steuber et al., 1997). The membrane-bound NqrB and C subunits each carry one covalently linked FMN which can be visualized by fluorography (Vorburger et al., 2016). Mrp is widely distributed among bacteria and archaea, especially among the organisms that need to adapt to elevated salt and/or high $\mathrm{pH}$ environments (Swartz et al., 2005; Dzioba-Winogrodzki et al., 2009). The Mrp from $V$. cholerae contains six subunits and catalyzes the electrogenic efflux of cytoplasmic $\mathrm{Na}^{+}, \mathrm{Li}^{+}$, and $\mathrm{K}^{+}$ions outward in a coupled reaction that transports external $\mathrm{H}^{+}$inward (Swartz et al., 2005; Dzioba-Winogrodzki et al., 2009). It is suggested, that the Mrp is needed for maximum protection of $V$. cholerae cells against alkali cations in neutral and alkaline environments. Furthermore, Mrp protects the $\mathrm{Na}^{+}$sensitive E. coli EP432 from high concentrations of toxic $\mathrm{Li}^{+}$(Dzioba-Winogrodzki et al., 2009).

In this study, we demonstrate that $V$. natriegens is a suitable expression host for functional membrane proteins. It is also an attractive non-pathogenic Vibrio strain for studying functions of proteins from pathogenic Vibrio species.

\section{RESULTS}

\section{Detection of Subunit NqrA of NQR-ST Complex Expressed in V. natriegens by Immune Staining After Western Blotting}

So far, expression of NQR from $V$. cholerae was only reported in its endogenous host. Using an expression vector conferring an N-terminal Strep-Tag to the cytoplasmic NqrA subunit, production of $V$. cholerae NQR produced in $V$. natriegens was confirmed with western blotting followed by immune detection (Figure 1A). Strep-tagged NqrA with an apparent molecular mass of $53.5 \mathrm{kDa}$ was detected in membranes from $V$. natriegens cells transformed with $\mathrm{pNqrST}$ and with purified NQR-ST complex, but not in membranes from $V$. natriegens cells transformed with the control vector, pBAD-TOPO. In all three samples, we observed an additional immunogenic protein with an apparent mass of $58 \mathrm{kDa}$, which probably represents a biotin-containing protein of the Vibrio expression hosts (Halang et al., 2013). Note that subunit NqrA is prone to proteolytic degradation (Vohl et al., 2014), resulting in an additional band below NqrA which was detected by immunostaining of the purified NQR-ST complex.

As previously reported for the NQR complex carrying a Histag at the N-terminus of subunit NqrA (Tao et al., 2008; Steuber et al., 2014b), the purified NQR-ST complex with additional Strep-Tag contained all six expected subunits, as confirmed by SDS-PAGE (Figure 1B). To separate the hydrophobic NqrB and NqrC subunits the SDS-PAGE was performed in the presence of $6 \mathrm{M}$ urea (Hayashi et al., 2001). Note that the small, hydrophobic NqrD and NqrE subunits stain only weakly with Coomassie.

\section{Detection of Subunits NqrB and NqrC of NQR-ST Complex Expressed in $V$. natriegens by in gel Fluography}

The subunits $\mathrm{NqrB}$ and $\mathrm{NqrC}$ exhibit fluorescence due to their covalently bound flavins (Hayashi et al., 2001). After separation of membranes from $V$. natriegens cells transformed with pNqrST or the control vector on SDS-PAGE without urea, we observed a single fluorescent band in both cases (Figure 2A). Subunits NqrB and NqrC co-migrate under these conditions. Fluorescence intensity was increased in the NQR-ST membranes when compared to membranes from control membranes, in accord with the expected overproduction. The weaker signal in control membranes resulted from the endogenous NqrB (accession number UNIPROT: A0A1B1EED7) and NqrC (accession number UNIPROT: A0A1B1EEE9) subunits from the NQR coded on region 455785-461628 of chromosome 1 from $V$. natriegens. These results were also confirmed by mass spectrometry analysis (Table 1 and Supplementary Table S1). As a positive control exhibiting flavin fluorescence, the purified NqrC' subunit was used comprising the covalently attached FMN but lacking the N-terminal transmembrane helix 
(Vohl et al., 2014). Accordingly, the protein runs at a lower apparent mass in SDS-PAGE compared to native NqrC. The purity of the control NqrC' was demonstrated by SDS-PAGE and Coomassie staining (Figure 2B).

\section{$\mathrm{Ag}^{+}$Inhibits NADH:Quinone Oxidoreduction Activity of $V$. natriegens pNqrST Membranes}

The inhibition of the NQR from $V$. cholerae with nanomolar concentrations of $\mathrm{Ag}^{+}$is a typical property of this enzyme (Steuber et al., 1997). Silver ions specifically and irreversibly inactivate the NQR by binding to the NADH-oxidizing NqrF subunit of the complex. With membranes from $V$. natriegens pNqrST, the oxidation of NADH with varying amounts of $\mathrm{AgNO}_{3}$ was examined (Figure 3). Without inhibitor, a specific activity of $\sim 1600 \mathrm{nmol} \mathrm{min} \mathrm{mg}^{-1}$ was observed, which decreased with increasing concentrations of silver nitrate. $\mathrm{Ag}^{+}$

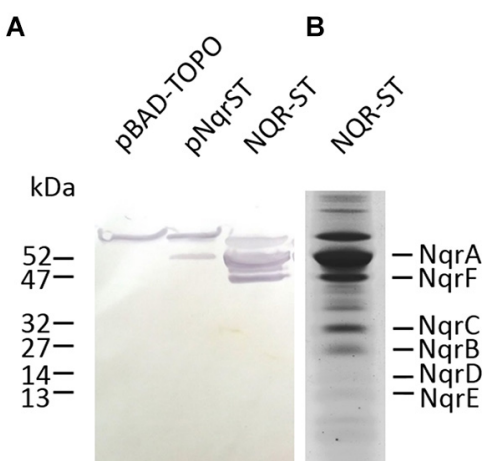

FIGURE 1 | Detection of NQR by immunostaining of Strep-NarA. After protein expression, the membranes from Vibrio natriegens pNarST were isolated and loaded onto SDS-PAGE. (A) Proteins were transferred to a nitrocellulose membrane and NqrA fused to the Strep-tag was detected by a chromogenic reaction. Lane 1, membranes from $V$. natriegens PBAD-TOPO (100 $\mu \mathrm{g})$; lane 2, membranes from $V$. natriegens pNarST (100 $\mu \mathrm{g}$ ); lane 3, positive control (purified NQR-ST complex, $50 \mu \mathrm{g}$ ). (B) Detection of six Nar subunits in NQR-ST after separation on a 10\% SDS gel with $6 \mathrm{M}$ urea. Hydrophobic NqrD and NqrE stain poorly in Coomassie.

\section{A}

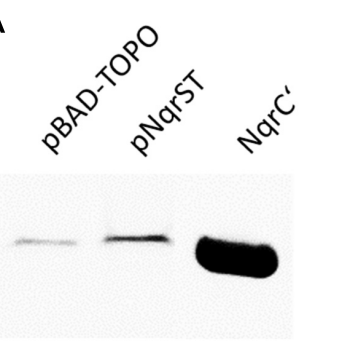

B

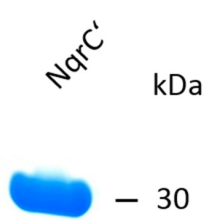

FIGURE 2 | Detection of the membrane-bound NarB and NarC subunits of $N Q R$ in $V$. natriegens membranes by in gel fluorography. Membranes from V. natriegens were separated on SDS-PAGE. (A) Lane 1, membranes from $V$. natriegens pBAD-TOPO $(100 \mu \mathrm{g})$; lane 2, membranes from $V$. natriegens pNarST (100 $\mu \mathrm{g})$; lane 3, positive control (NqrC', $40 \mu \mathrm{g})$. (B) SDS gel of the positive control (NarC') stained with Coomassie. in nanomolar concentration range (20-40 $\left.\mathrm{nM} \mathrm{AgNO}_{3}\right)$ led to a half-maximal inactivation of NADH oxidation activity. The membranes from $V$. natriegens $\mathrm{pBAD}$-TOPO also showed NADH oxidation activity, due to the endogenous NQR (Figure 4). Nevertheless, a significant lower activity with $\sim 950 \mathrm{nmol} \mathrm{min}^{-1}$ $\mathrm{mg}^{-1}$ was observed, compared to $V$. natriegens pNqrST. The NADH oxidation activity of membranes from $V$. natriegens pBAD-TOPO could also be inhibited with $\mathrm{Ag}^{+}$, but much higher concentrations of the inhibitor were needed, with half-maximal inactivation in the range of $0.2-0.3 \mu \mathrm{M} \mathrm{AgNO}$.

\section{Solubilized $V$. natriegens pNqrST Membranes Contain a Protein Complex With NADH Dehydrogenase Activity}

To further characterize the NADH dehydrogenase activity, solubilized membrane proteins were separated in a Blue Native PAGE (BN PAGE) followed by in gel activity staining to detect proteins with $\mathrm{NADH}$ dehydrogenase activity. Figure 5 shows the results for the membranes and solubilized membrane proteins of $V$. natriegens $\mathrm{pNqrST}$ and $V$. natriegens $\mathrm{pBAD}-\mathrm{TOPO}$, respectively. With the solubilized membranes, a band around $185 \mathrm{kDa}$ molecular weight was stained due to precipitation of nitro blue diformazan (Figure 5, boxes 1, 2), indicating NADH dehydrogenase activity. These bands showed the same apparent mass as the purified NQR complex exhibiting in gel activity which was used as control. Staining of the $V$. natriegens pBADTOPO band at $185 \mathrm{kDa}$ (Figure 5, box 2) was less intensive than with solubilized pNqrST membranes (Figure 5, box 1), indicating that endogenous NQR from $V$. natriegens was also solubilized with 1\% DDM and exhibited a similar migration behavior as the Strep-His-tagged NQR from $V$. cholerae. Mass spectrometry analysis confirmed the presence of all six Nqr subunits in these protein bands (Table 1 and Supplementary Table S1). We conclude that holo-NQR complex from $V$. cholerae was produced in $V$. natriegens. Furthermore, two sharp bands migrated roughly between the NQR complex $(185 \mathrm{kDa})$ and BSA $(66 \mathrm{kDa})$ in each solubilization sample (Figure 5, box 3/box 5, box 4/box 6), indicating the presence of smaller NQR sub-complexes. Mass spectrometry analysis identified NqrA and NqrC in both upper protein bands (Figure 5, boxes 3, 4). NqrA, NqrC and NqrF were identified in the lower protein band of solubilized $V$. natriegens pBAD-TOPO membranes (Figure 5, box 6). NqrA and NqrF were identified in the lower protein band of solubilized $V$. natriegens pNqrST membranes (Figure 5, box 5, Table 1 and Supplementary Table S1). The NADH oxidizing NqrF subunit could not be detected in the upper protein bands (Figure 5, boxes 3,4 ) by mass spectrometry, probably because of limited sensitivity. However, its presence is very likely since neither NqrA nor NqrC exhibit NADH dehydrogenase activity.

\section{Mrp Is Expressed in V. natriegens}

Like NQR, the mrp operon from $V$. cholerae encoded by the pVcMrp plasmid had not been expressed in $V$. natriegens before, so we first performed expression tests with different L-arabinose concentrations $(0.2,0.02$, and $0.002 \%)$ and various induction times. Cells broken by treatment with SDS sample buffer 
TABLE 1 | Identification of Nar subunits in V. natriegens PBAD-TOPO and Vibrio natriegens pNqr-ST by mass spectrometry.

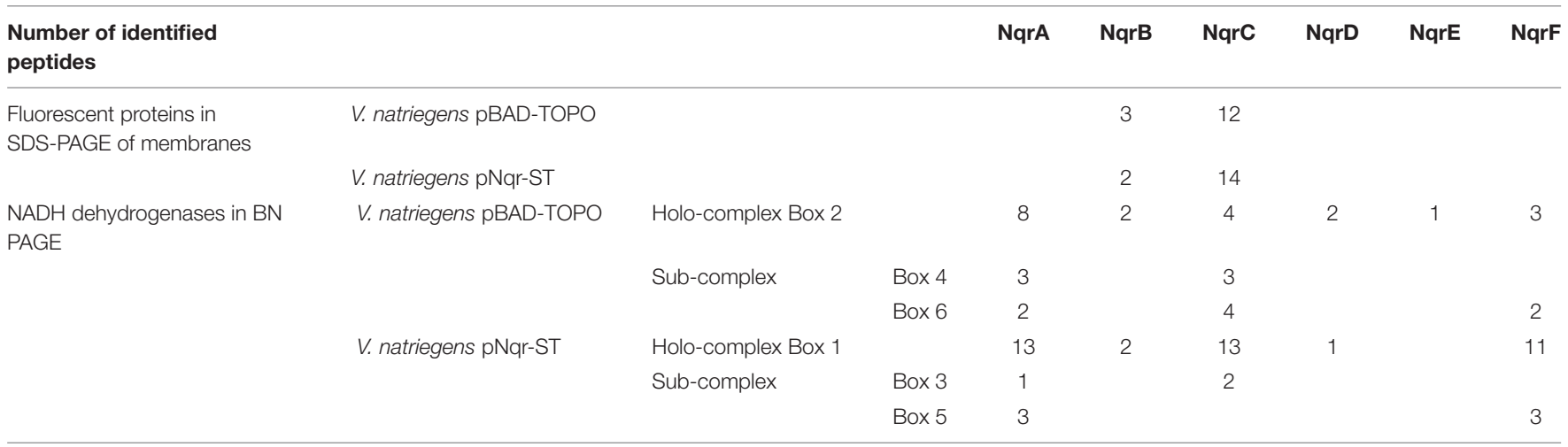

To confirm the presence of NqrB and NarC, fluorescent protein bands from membranes separated by SDS-PAGE were analyzed (Figure 2A). To describe the composition of NQR complexes, bands with NADH dehydrogenase activity after BN PAGE (indicated by boxes in Figure 5) were analyzed. The number of Nqr-derived peptides identified in each sample varies from 1 to 14. For peptide sequences see Supplementary Table S1.

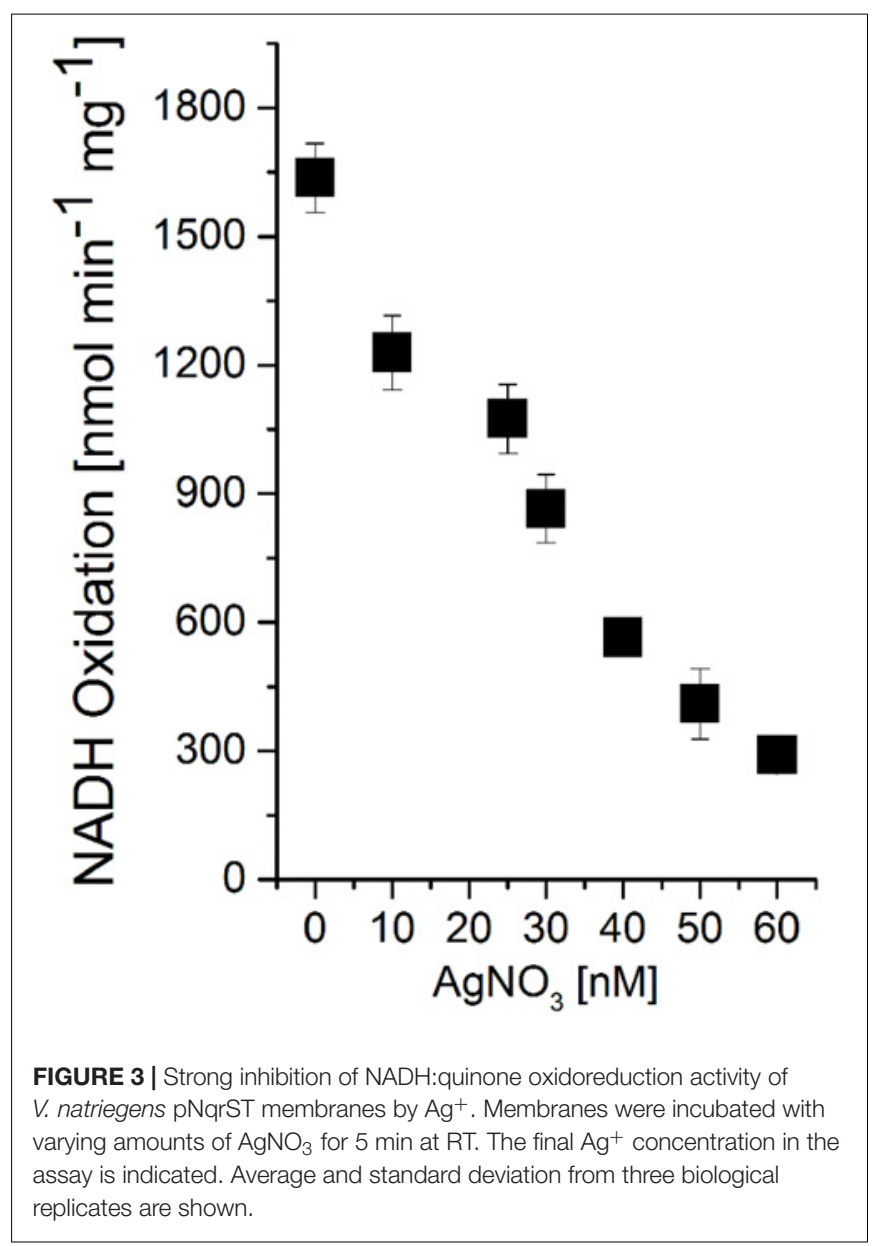

were analyzed by SDS-PAGE. MrpG was detected by western blotting and immune detection with anti-V5-HRP antibodies and chemiluminescence (apparent molecular mass of MrpG with $\mathrm{His}_{6}$ tag and V5-epitope tag: $13.5 \mathrm{kDa}$ ) (Figure 6). The strongest signal was observed with $0.02 \% \mathrm{~L}$-arabinose after $2 \mathrm{~h}$ of induction. Up to $2 \mathrm{~h}$ induction, no MrpG-V5 was detected. After

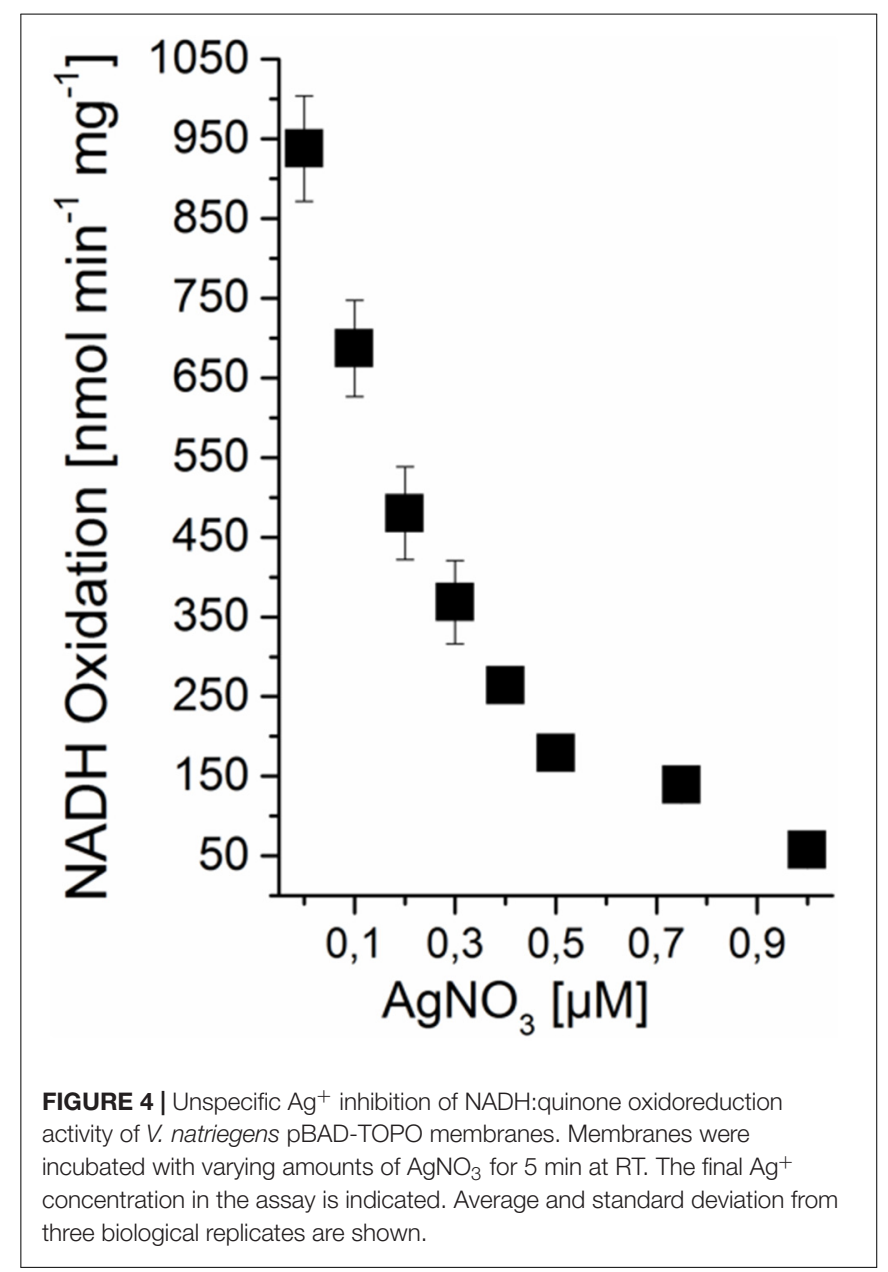

$2 \mathrm{~h}$ induction time, the expression levels declined continuously. Furthermore, three protein bands with a higher molecular weight became visible in every crude extract sample, even in the negative control where no L-arabinose was added. These proteins were already visible after $30 \mathrm{~s}$ of exposure (data not shown), whereas the MrpG-V5 protein band required 2 min exposure 


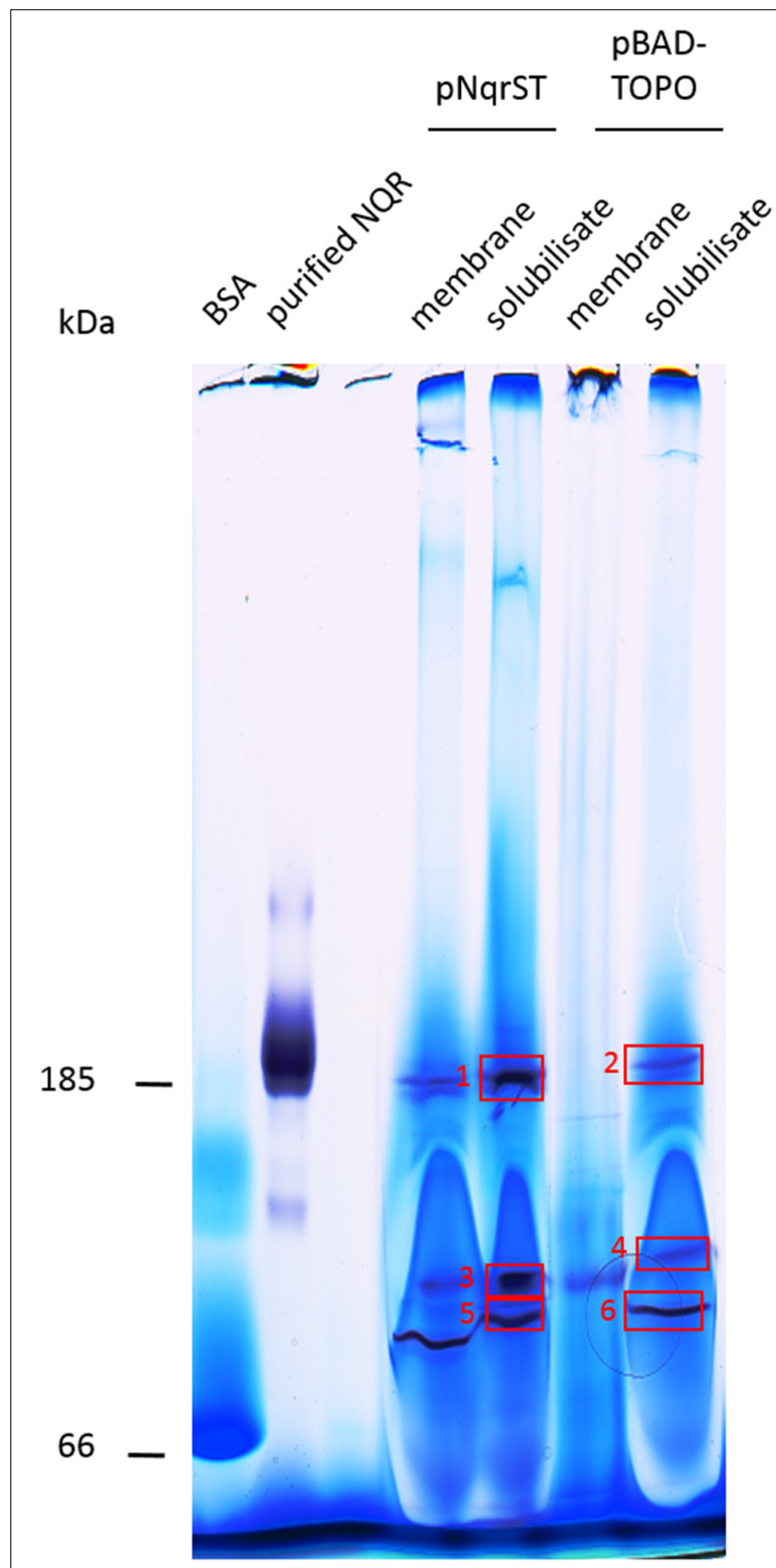

FIGURE 5 | Blue native PAGE of $V$. natriegens membranes and detection of $\mathrm{NADH}$ dehydrogenase activity. Membranes and membranes solubilized with $1 \%$ DDM were separated by BN PAGE with a gradient from 4 to $16 \%$ acrylamide in the gel (100 $\mu \mathrm{g}$ solubilized membrane, or $200 \mu \mathrm{g}$ membrane, per lane). Protein bands, which contained NADH dehydrogenase complexes or sub-complexes thereof, were identified by in gel activity stain. Boxes indicate proteins subjected to mass spectrometry analysis. Upper band represents holo-NQR, the lower bands a sub-complex of NqrA, NqrF, and NarC (Table 1 and Supplementary Table S1). Negative control, BSA $(50 \mu \mathrm{g})$. Positive control, purified His-tagged NQR $(4 \mu \mathrm{g})$.

time. Membranes from $V$. natriegens $\mathrm{pVc}-\mathrm{Mrp}$ and $V$. natriegens pBAD-TOPO were also analyzed in a western blot. Only the $V$. natriegens pVc-Mrp membranes showed the expected band at $\sim 13 \mathrm{kDa}$, indicating the presence of MrpG-V5 in V. natriegens membranes.

\section{Functional Mrp Permits Growth of V. natriegens at High $\mathrm{Li}^{+}$Concentrations}

Dzioba-Winogrodzki et al. (2009) postulated that the Mrp from $V$. cholerae is a cation $/ \mathrm{H}^{+}$antiporter, protecting the $\mathrm{Na}^{+}$sensitive strain E. coli EP432 from stress caused by high external concentrations of $\mathrm{Li}^{+}$. To analyze if Mrp also protects $V$. natriegens from high $\mathrm{Li}^{+}$concentrations, growth experiments in glass test tubes were performed. First, the critical $\mathrm{Li}^{+}$concentration preventing growth of the $V$. natriegens strains was determined (Figure 7). V. natriegens pVc-Mrp and $V$. natriegens pBAD-TOPO grown in LBS medium showed no significant differences in the optical densities after $20 \mathrm{~h}$ of incubation without added $\mathrm{Li}^{+}$. In presence of $300 \mathrm{mM}$ or $400 \mathrm{mM} \mathrm{LiCl}, V$. natriegens pVc-Mrp showed a much higher optical density compared to $V$. natriegens pBAD-TOPO after 20 h. With $300 \mathrm{mM} \mathrm{LiCl}$ added, V. natriegens $\mathrm{pVc}-\mathrm{Mrp}$ reached a final $\mathrm{OD}_{600}$ of $1.4 \pm 0.04$, whereas $V$. natriegens pBADTOPO had a final $\mathrm{OD}_{600}$ of $0.25 \pm 0.04$. With $400 \mathrm{mM} \mathrm{LiCl}$, $V$. natriegens $\mathrm{pVc}-\mathrm{Mrp}$ reached a final $\mathrm{OD}_{600}$ of $1.02 \pm 0.02$ and $V$. natriegens $\mathrm{pBAD}-\mathrm{TOPO}$ had a final $\mathrm{OD}_{600}$ of $0.12 \pm 0.01$. We conclude that Mrp permits the growth of $V$. natriegens at high $\mathrm{Li}^{+}$concentrations, which inhibits the growth of $V$. natriegens pBAD-TOPO. Cells grown in presence of different $\mathrm{KCl}$ concentrations were used as a control, revealing no toxic effect of $\mathrm{K}^{+}$. Therefore, the observed inhibition of $V$. natriegens pBAD-TOPO by LiCl was not caused by elevated chloride concentrations.

We also monitored growth of $V$. natriegens pVc-Mrp in presence of $400 \mathrm{mM} \mathrm{LiCl}$ over time with a plate reader (Figure 8). Growth of the $V$. natriegens pBAD-TOPO reference strain was followed in parallel. Again, the strains exhibit different growth phenotypes. Although both strains reached nearly the same final $\mathrm{OD}_{600}$ of $0.85 \pm 0.13$ (pBAD-TOPO) and $0.87 \pm 0.04$ (pVc-Mrp), $V$. natriegens pVc-Mrp reached its highest cell density of $0.9 \pm 0.03$ already after $17 \mathrm{~h}$, compared to $24 \mathrm{~h}$ with $V$. natriegens pBAD-TOPO (Figure 8). Aeration of cultures is improved in microtiter plates compared to glass tubes, and we conclude that the $V$. cholerae Mrp in its active state protected $V$. natriegens from toxic $\mathrm{LiCl}$ under both conditions.

\section{DISCUSSION}

The expression of multisubunit membrane proteins faces two major problems. First, the coordinated expression of the subunits and second, the correct assembly and insertion of the complex into the membrane. Membrane protein complexes normally cannot be reconstituted by expression of the subcomplexes only. They need, e.g., interaction partners for stable folding and function (Zorman et al., 2015). Until now, this problem was solved mainly with co-expression of multiple genes using, e.g., the Duet (Novagen) vectors (Tolia and Joshua-Tor, 2006). These vectors have multiple cloning sites, five origins of 


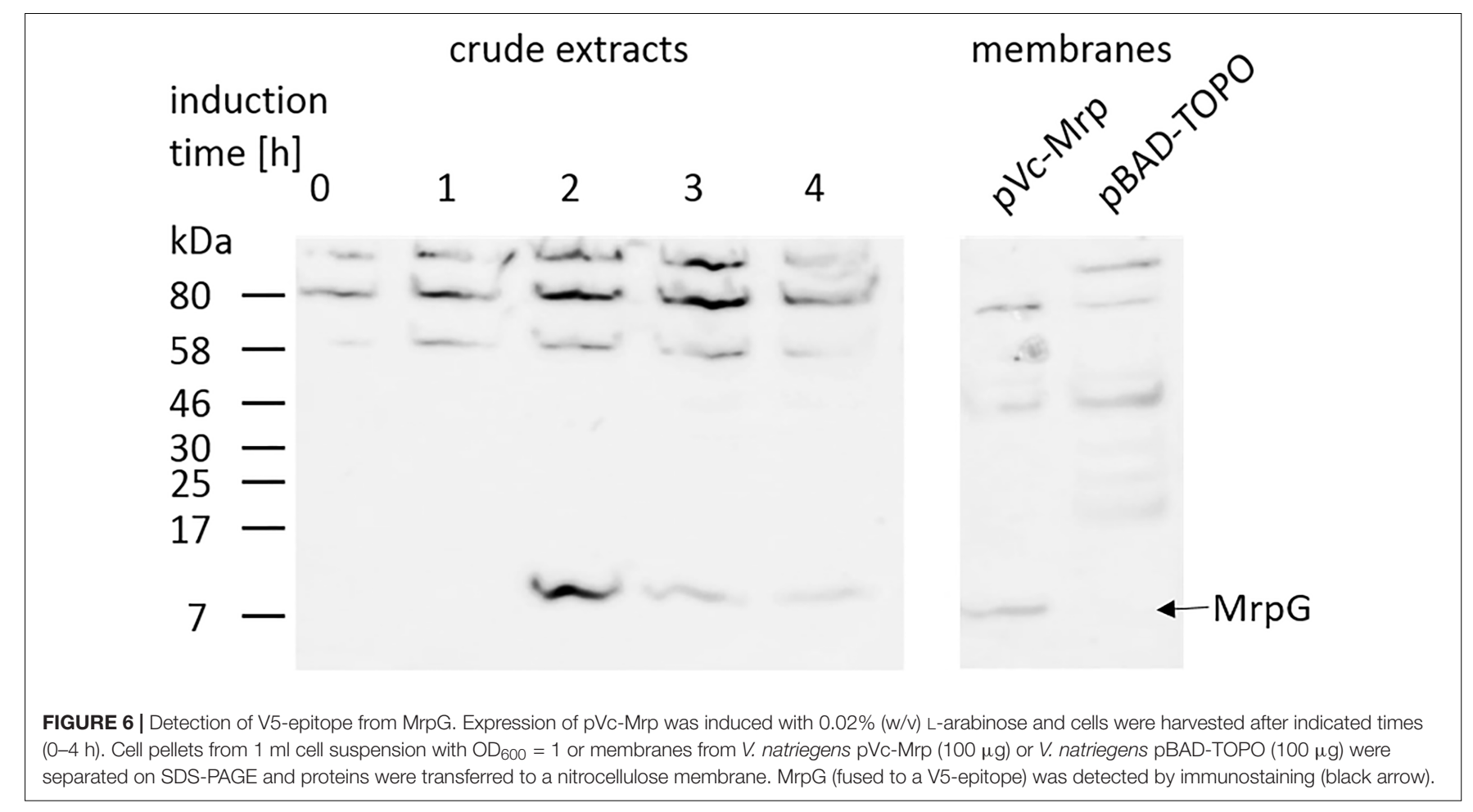

replication and four antibiotic selection markers, allowing the simultaneous expression of up to eight proteins (Tolia and Joshua-Tor, 2006). The MultiBac system is another tool (Pelosse et al., 2017). It is a baculovirus/insect expression system specifically engineered for production of functional multiprotein complexes (Berger et al., 2004; Fitzgerald et al., 2006; Pelosse et al., 2017). In this study, we were able to express two membrane-bound protein complexes in $V$.natriegens by introducing the structural operons into vectors, which did not code for additional assembly factors. In case of the NQR, a functional protein complex could be produced without coexpression of $a p b E$ or nqrM. ApbE is a flavin transferase catalyzing the covalent attachment of FMN to NqrB and NqrC in V. cholerae (Bertsova et al., 2013). It is necessary for NQR maturation, since the FMNs are required for the transfer of the electrons through the NQR complex. NqrM was predicted to deliver Fe to subunits NqrD and NqrE (Kostyrko et al., 2015). Subunits NqrD and NqrE harbor an iron cluster located in the midst of the membrane part of NQR which is proposed to participate in intramolecular electron transfer (Steuber et al., 2014b). V. natriegens was able to produce active NQR, probably because it harbors an endogenous NQR. As in $V$. cholerae, $a p b E$ and $n q r M$ are located immediately downstream of the nqr operon in $V$. natriegens. The genes $n q r M$ (accession number UNIPROT: A0A1B1EEC2) and $a p b E$ (accession number UNIPROT: A0A1B1EEE5) from $V$. natriegens are homologous to nqrM (accession number UNIPROT: Q9KPS4) and $a p b E$ (accession number UNIPROT: A5F5Y3) in $V$. cholerae. Both organisms contain two chromosomes. The apbE, nqrM and the nqr genes are located on the larger chromosome (Supplementary Figure S1). Our study shows that

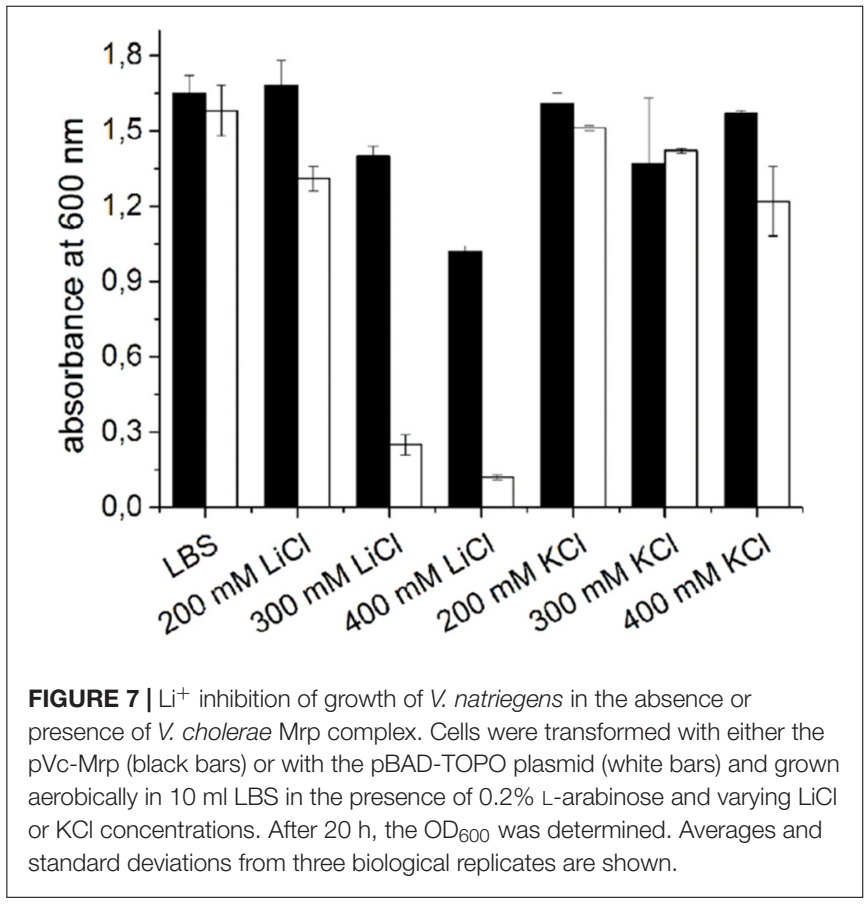

ApbE and probably, NqrM, from $V$. natriegens function in maturation of the $V$. cholerae NQR.

Furthermore, we could detect the holo NQR by NBT staining in a BN PAGE. Here a signal at around $185 \mathrm{kDa}$ became visible in the solubilisates (Figure 5, boxes 1,2) and all NQR subunits could be identified by mass spectrometry. Also at a lower molecular 


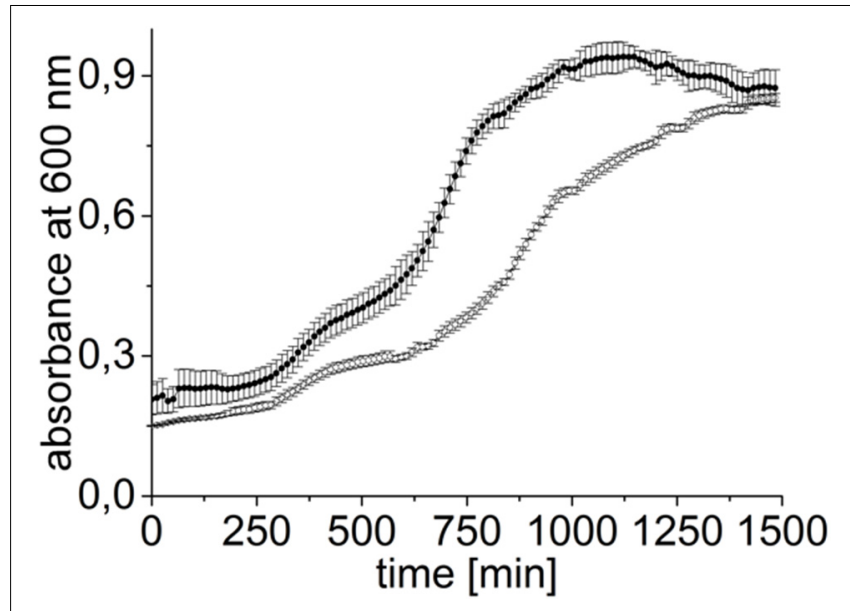

FIGURE 8 | Mrp expression confers a growth advantage to $V$. natriegens at high $\left[\mathrm{Li}^{+}\right]$. Cells were transformed with either the pVc-Mrp (closed circles) or with the PBAD-TOPO plasmid (empty circles) and grown aerobically in LBS in the presence of $0.2 \% \mathrm{~L}$-arabinose and $400 \mathrm{mM} \mathrm{LiCl}$ at $37^{\circ} \mathrm{C}$ in a 96 -well plate. Averages and standard deviations from three biological replicates are shown.

weight level, two signals became visible in each solubilisate sample, indicating two sub-complexes (Figure 5, boxes 3-6). Mass spectrometry identified NqrA and $\mathrm{NqrC}$ in the upper protein bands (Figure 5, boxes 3,4) and NqrA, NqrC, and NqrF (Figure 5, box 6) or NqrA and NqrF (Figure 5, box 5) in the lower ones. We suggest that the upper protein band comprises an Nqr sub-complex of NqrABCF. The formation of such a subcomplex was observed before during purification of the NQR (Tao et al., 2008). It is possible, that NqrF and NqrB, which were not identified in these samples, were washed out before mass spectrometry. When the BN PAGE gel is stained with NBT and not fixed with TCA (trichloroacetic acid) we observed that the proteins are washed out over time and only the purple precipitate remains in the gel matrix. Therefore, we recommend to prepare two BN PAGEs in parallel: One is stained with NBT and the other one is treated with 20\% TCA for $30 \mathrm{~min}$ and afterward with Coomassie. By comparison of the two gels, the corresponding protein bands are recovered from the TCA-fixed gel for mass spectrometry. The observed depletion of proteins from BN PAGE also offers a rationale for the fact that the upper band stained with NBT apparently obtained only NqrA and NqrC, but not NqrF which represents the only NADH oxidizing subunit in the NQR complex.

While NQR is a primary $\mathrm{Na}^{+}$pump, Mrp represents a secondary transporter catalyzing cation $/ \mathrm{H}^{+}$antiport. Like NQR, it is a membrane-bound protein complex composed of six subunits (Swartz et al., 2005; Dzioba-Winogrodzki et al., 2009; Morino et al., 2014, 2017). Functional expression of the Mrp was confirmed by in vivo growth experiment demonstrating the rescue of cell growth despite high (toxic) amounts of $\mathrm{Li}^{+}$. There was an optimum of expression after $2 \mathrm{~h}$ of incubation with $0.02 \%$ L-arabinose, followed by subsequent decrease of expression levels. This could reflect increased proteolysis of Mrp, or a decrease in expression levels. We favor the latter explanation because we observed large variations in the capacity of $V$. natriegens transformed with pVc-Mrp to survive high $\left[\mathrm{Li}^{+}\right]$. In all experiments, $\mathrm{pVc}$-Mrp clearly conferred a growth advantage compared to the empty vector, but the final cell yields observed with pVc-Mrp varied between $\mathrm{OD}_{600}=1$ at $300 \mathrm{mM} \mathrm{LiCl}$ and $\mathrm{OD}_{600}=0.5$ at $400 \mathrm{mM} \mathrm{LiCl}$. These variations most likely are caused by L-arabinose isomerase (accession number UNIPROT: A0A1B1EI15), an enzyme allowing degradation of the inducer L-arabinose by $V$. natriegens.

In summary, $V$. natriegens is an attractive expression host because of its very high growth rate, its non-pathogenicity, and because it allows scalable expressions of large membrane proteins. Once the metabolism of L-arabinose is blocked by future genetic engineering (Guzman et al., 1995; Baba et al., 2006) $V$. natriegens could become an alternative to E. coli as expression host, especially for the overproduction of proteins from pathogenic bacteria.

\section{MATERIALS AND METHODS}

\section{Bacterial Strains and Plasmids}

Vibrio natriegens ATCC 14048 was transformed with the plasmids pBAD-TOPO (Invitrogen pBAD-TOPO ${ }^{\circledR}$ TA), pVcMrp (Dzioba-Winogrodzki et al., 2009) or pNqrST (this work) according to the protocol of Weinstock et al. (2016). E. coli Top 10 (Invitrogen) was used for the cloning of the pNqrST plasmid.

\section{Construction and Transformation of Vector pNqrST}

The plasmid was constructed for the expression of the NQR from $V$. cholerae carrying a polyhistidine tag and a Strep-Tag at the N-terminus of subunit NqrA. The plasmid is based on the pNQR1 plasmid (Tao et al., 2008) containing the whole $n q r$ operon, which consists of the six genes nqrABCDEF and a sequence encoding for six histidine residues ( $\mathrm{His}_{6}$-Tag) fused to the $5^{\prime}$ end of the nqrA gene. For the pNqrST construct, the fragment coding for the $\mathrm{His}_{6}$-Tag in pNQR1 and a part of the upstream $a r a B$ region were cut out from pNQR1 with the restriction enzymes SgrAI and NdeI. The linearized and truncated pNQR1 plasmid was then ligated with a synthetic SgrAI - NdeI DNA fragment (Eurofins Genomics). This fragment comprises the lost $a r a B$ region, a $\mathrm{His}_{6}$-Tag and two streptavidin polypeptides (Supplementary Figure S2). This results in NQR complex with subunit NqrA carrying, at the N-terminus, a His $6^{-}$ Tag followed by two consecutive Strep-Tags. Note that pNqrST also confers a protease restriction site (preScission site) for the HRV 3C protease between the histidine residues and the StrepTag. Correct cloning was confirmed by sequencing (Eurofins Genomics).

Chemo-competent E. coli Top 10 cells were prepared following the protocol of (Inoue et al., 1990). For transformation, an aliquot of $100 \mu \mathrm{l}$ competent E. coli cells was thawed on ice and mixed with $250 \mathrm{ng}$ pNqrST. After incubation on ice for $30 \mathrm{~min}$, cells were heat shocked at $42^{\circ} \mathrm{C}$ for $30 \mathrm{~s}$ and cooled for $5 \mathrm{~min}$ on ice. Then $900 \mu \mathrm{l}$ SOC medium (20 g/l tryptone, $5 \mathrm{~g} / \mathrm{l}$ yeast extract, $10 \mathrm{mM} \mathrm{NaCl}, 2.5 \mathrm{mM} \mathrm{KCl}, 10 \mathrm{mM} \mathrm{MgCl}_{2}, 10 \mathrm{mM}$ 
$\mathrm{MgSO}_{4}$, and $20 \mathrm{mM}$ glucose, $\mathrm{pH}$ adjusted to 7.0 with $\mathrm{NaOH}$ ) was added. After incubation at $37^{\circ} \mathrm{C}$ for $1 \mathrm{~h}$ the cell suspension was centrifuged for $1 \mathrm{~min}$ with $11000 \mathrm{~g}$ and $900 \mu \mathrm{l}$ of the supernatant were discarded. The cell pellet was resuspended in the remaining $100 \mu \mathrm{l} \mathrm{SOC}$ medium and cells were distributed on LB agar plates supplemented with $100 \mu \mathrm{g} / \mathrm{ml}$ ampicillin. Positive clones were identified after plasmid preparation (Miniprep Kit II, peqlab) followed by restriction analyses.

\section{Growth of $V$. natriegens}

Cells were cultivated in LBS medium ( $10 \mathrm{~g} / \mathrm{l}$ tryptone, $5 \mathrm{~g} / \mathrm{l}$ yeast extract, $375 \mathrm{mM} \mathrm{NaCl}, 4.2 \mathrm{mM} \mathrm{KCl}$, and $23.1 \mathrm{mM} \mathrm{MgCl}_{2}$ ). To obtain a preculture $10 \mathrm{ml}$ LBS medium with $100 \mu \mathrm{g} / \mathrm{ml}$ ampicillin was inoculated with a single colony and cultivated at $30^{\circ} \mathrm{C}$ on a rotary shaker at $180 \mathrm{rpm}$ overnight. With this preculture $1 \mathrm{~L}$ medium was inoculated (1:100) and incubated at $37^{\circ} \mathrm{C}$ with moderate aeration. At $\mathrm{OD}_{600} \sim 0.8 \mathrm{~L}$-arabinose was added to a final concentration of $0.02 \%$ to induce the protein expression. $V$. natriegens was stored at $-80^{\circ} \mathrm{C}$ as $15 \%$ glycerol stock.

\section{Membrane Isolation}

Cells were harvested after 1-2 $\mathrm{h}$ of incubation with L-arabinose by centrifugation at $6000 \mathrm{~g}$ for $30 \mathrm{~min}$. Pellets were resuspended in cell lysis buffer $(500 \mathrm{mM} \mathrm{KCl}, 20 \mathrm{mM}$ potassium phosphate buffer $\mathrm{pH} 8$ ) at a concentration of $0.2 \mathrm{~g} / \mathrm{ml}$ and supplemented with protease inhibitor (cOmplete ULTRA Tablets, Roche Diagnostics), $1 \mathrm{mM} \mathrm{DTT,} 0.5 \mathrm{mM} \mathrm{MgCl} 2$ and a trace of DNase I. The cells were disrupted in a continuous cell lysis system at $20 \mathrm{kPsi}$ (Emulsiflex C3, Avestin). The suspension was centrifuged with $27000 \mathrm{~g}$ for $30 \mathrm{~min}$ to remove the unbroken cells and cell debris. The supernatant was centrifuged with $250000 \mathrm{~g}$ for $1 \mathrm{~h}$ to collect the membranes. The pellet was resuspended in buffer A [50 mM potassium phosphate buffer $\mathrm{pH} 7.5,5 \%$ glycerol $(\mathrm{w} / \mathrm{v}), 300 \mathrm{mM} \mathrm{KCl}$ ] followed by another centrifugation step in buffer $\mathrm{A}$ to wash the membranes. The membranes were resuspended in $5 \mathrm{ml}$ buffer $\mathrm{A}$ and frozen in liquid $\mathrm{N}_{2}$.

\section{In gel Fluorography}

Fluorescence of covalently bound flavins in $\mathrm{NqrB}$ and $\mathrm{NqrC}$ was detected using the ImageQuant LAS 4000 imager $\left(\lambda_{\text {excitation }}=460\right.$, emission filter $=$ Y515 CyTM2 $)$. As a positive control the purified $\mathrm{NqrC}^{\prime}$ subunit was used. This protein is a truncated variant of the $\mathrm{NqrC}$ subunit of the $V$. cholerae NQR comprising the covalently attached FMN but lacking the N-terminal transmembrane helix (Vohl et al., 2014). The molecular mass of $\mathrm{NqrC}^{\prime}$ is $25.38 \mathrm{kDa}$.

\section{PAGE and Activity Staining}

Denaturing polyacrylamide gel electrophoresis (SDS-PAGE) was performed with a $12 \%$ polyacrylamide gel (Schägger and von Jagow, 1987). Protein and membrane suspensions were diluted in $5 \times$ SDS sample buffer [500 mM DTT, $1 \mathrm{M}$ Tris-HCl pH $6.8,5 \%(\mathrm{w} / \mathrm{v})$ SDS, $28.8 \%$ glycerol, bromophenol blue]. Cell pellets were resuspended in $50 \mu \mathrm{l} 1 \times$ SDS sample buffer for cell disruption.
For the Blue native PAGE, SERVAGel ${ }^{\mathrm{TM}}$ Native Gels (SERVA) with a gradient from 4 to $16 \%$ acrylamide were used. The electrophoresis was performed following the instructions of the manufacturer, modified as follows. First, the gel was run with $30 \mathrm{~V}$ for $\sim 3 \mathrm{~h}$. Then the voltage was increased to $100 \mathrm{~V}$ for $\sim 1.5 \mathrm{~h}$. When $1 / 3-1 / 2$ of the electrophoresis was completed the blue $1 \times$ cathode buffer $[50 \mathrm{mM}$ Tricine, $15 \mathrm{mM}$ Bis-Tris $\mathrm{pH}$ 7.0, $0.002 \%(\mathrm{w} / \mathrm{v})$ Coomassie Blue] was changed to $1 \times$ cathode buffer (50 mM Tricine, $15 \mathrm{mM}$ Bis-Tris $\mathrm{pH}$ 7.0) without Coomassie. Anode buffer remained unchanged $(50 \mathrm{mM}$ Bis-Tris $\mathrm{pH}$ 7.0). Afterward, the voltage was increased again to $200 \mathrm{~V}$ until the blue front reached the bottom of the gel. Activity staining to detect $\mathrm{NADH}$ oxidizing proteins was conducted according to the protocol of Yan et al. (2007). Photographs of the gel were taken before activity staining and $30 \mathrm{~min}$ or $20 \mathrm{~h}$ after incubation in staining solution $[50 \mathrm{mM}$ potassium phosphate buffer $\mathrm{pH} 7.0,0.2 \mathrm{mg} / \mathrm{mL}$ Nitro blue tetrazolium chloride (NBT), $\left.0.1 \mathrm{mg} / \mathrm{mL} \mathrm{Na} \mathrm{N}_{2} \mathrm{NADH}\right]$. The purified Histagged NQR complex (Tao et al., 2008) served as positive control.

\section{Mass Spectrometry}

Nano-LC-ESI-MS/MS experiments were performed on an EASYnLC 1200 system (Thermo Fisher Scientific, Germany) coupled to a LTQ-Orbitrap XL hybrid mass spectrometer (Thermo Fisher Scientific, Germany) using an EASY-Spray nanoelectrospray ion source (Thermo Fisher Scientific, Germany). Tryptic peptides were directly injected to an EASY-Spray analytical column (PepMap RSLC C18, $2 \mu \mathrm{m} 100 \AA 50 \mu \mathrm{m} \times 250 \mathrm{~mm}$ column, Thermo Fisher Scientific, Germany) operated at constant temperature of $40^{\circ} \mathrm{C}$. Gradient elution was performed at a flow rate of $250 \mathrm{nl} / \mathrm{min}$ using a $30 \mathrm{~min}$ gradient from 2 to $40 \%$ solvent B in $30 \mathrm{~min}$, from 40 to $75 \%$ in $5 \mathrm{~min}$ and final from 75 to $95 \%$ in $10 \mathrm{~min}$ or rather a $60 \mathrm{~min}$ gradient from 2 to $45 \%$ in $60 \mathrm{~min}$, in $5 \mathrm{~min}$ to $95 \%$. Solvents used were $0.1 \%$ formic acid (solvent A) and $80 \%$ acetonitrile $/ 0.1 \%$ formic acid (end concentration) (solvent B). The LTQ-Orbitrap was operated under the control of Xcalibur software (version 2.1, Thermo Fisher Scientific Inc., United States). Survey spectra $(\mathrm{m} / \mathrm{z}=200-2000)$ were detected in the Orbitrap at a resolution of 60,000 at $\mathrm{m} / \mathrm{z}=400$. Data dependent tandem mass spectra were generated for the seven most abundant peptide precursors in the linear ion trap. For all measurements using the Orbitrap detector, internal calibration was performed using lock-mass ions from ambient air as described in Olsen et al. (2005). The data were analyzed by Mascot 2.6 (Matrix Science, United Kingdom), which was used as search engine for protein identification. Spectra were searched against the $V$. natriegens subset of the NCBI protein sequence database downloaded as FASTAformatted sequences (https://www.ncbi.nlm.nih.gov/, update from December 2016/May 2017). Search parameters specified trypsin as cleaving enzyme, allowing three missed cleavages, a $5 \mathrm{ppm}$ mass 32 tolerance for peptide precursors and $0.6 \mathrm{Da}$ tolerance for fragment ions. Methionine oxidation was allowed as variable modification and carbamidomethylation 
of cysteine residues was set as fixed modification. The Mascot results were transferred to Scaffold ${ }^{\mathrm{TM}}$ Software 4.6.1 (Proteome Software, United States).

\section{Immune Detection}

After separation, proteins were transferred to a nitrocellulose membrane by western blotting (Mini Trans-Blot ${ }^{\circledR}$ Cell system from Bio-Rad). The membrane was incubated for $1 \mathrm{~h}$ in blocking solution [137 mM NaCl, $2.7 \mathrm{mM} \mathrm{KCl,} 1.8 \mathrm{mM} \mathrm{KH}_{2} \mathrm{PO}_{4}, 10 \mathrm{mM}$ $\mathrm{Na}_{2} \mathrm{HPO}_{4}, 0.05 \%$ Tween, and 5\% (w/v) skimmed milk powder]. After two washing steps with PBST (137 mM NaCl, $2.7 \mathrm{mM}$ $\mathrm{KCl}, 1.8 \mathrm{mM} \mathrm{KH}_{2} \mathrm{PO}_{4}, 10 \mathrm{mM} \mathrm{Na}_{2} \mathrm{HPO}_{4}$, and $0.05 \%$ Tween) for $5 \mathrm{~min}$ the nitrocellulose membrane was incubated for $1 \mathrm{~h}$ with antibodies against V5-MrpG, or Strep-NqrA, which were diluted 1:5000 in blocking solution. In both cases HRP-conjugated antibodies were used (V5 Tag Monoclonal Antibody E10/V4RR HRP, Thermo Fisher Scientific; Strep-Tactin ${ }^{\circledR}$ conjugated to horseradish peroxidase, iba). After two washing steps with PBST, bound antibodies were detected using chemiluminescence Clarity $^{\mathrm{TM}}$ Western ECl Blotting Substrates (Bio-Rad). Exposure (2 min) and detection were performed with the Image Quant LAS 400 (GE Healthcare). For a chromogenic immunodetection, the membrane was incubated with $20 \mathrm{ml} \mathrm{PBST,} 200 \mu \mathrm{l} 3 \% 4$ Chloro-1-naphthol, and $20 \mu \mathrm{l} 30 \% \mathrm{H}_{2} \mathrm{O}_{2}$. As positive control for the immunostaining of Strep-NqrA, the holo NQR-ST complex expressed in $V$. cholerae from the pNqrST plasmid was used. The Strep-His-tagged NQR complex was purified by Nickel affinity chromatography as described for His-tagged NQR complex (Tao et al., 2008).

\section{Enzyme Kinetics}

$\mathrm{NADH}$ oxidation assays were carried out under stirring in a quartz cuvette $\left(1 \mathrm{~cm}\right.$ diameter) in a total volume of $1 \mathrm{ml}$ at $25^{\circ} \mathrm{C}$ using a Diode-Array Spectrophotometer. NADH oxidation was followed at $340 \mathrm{~nm}\left(\varepsilon_{\mathrm{NADH}}=6.22 \mathrm{mM}^{-1}\right)$ (Juárez et al., 2009). To avoid precipitation of $\mathrm{AgCl}, \mathrm{NQR}$ activity in the presence of $\mathrm{Ag}^{+}$was measured in reaction buffer containing $20 \mathrm{mM}$ Tris $\mathrm{H}_{2} \mathrm{SO}_{4} \mathrm{pH} 8,100 \mathrm{mM} \mathrm{Na}_{2} \mathrm{SO}_{4}, 100 \mu \mathrm{M}$ ubiquinone, and $100 \mu \mathrm{M}$ $\mathrm{Na}_{2} \mathrm{NADH}$. A total of $20 \mu \mathrm{g}$ membranes were incubated with varying amounts of $\mathrm{AgNO}_{3}$ for $5 \mathrm{~min}$ at RT. Membrane aliquots (20 $\mu \mathrm{g}$ protein) were added to reaction buffer and the oxidation of NADH was followed.

\section{In vivo Function of Mrp Monitored in Growth Experiments}

Vibrio natriegens transformed with $\mathrm{pVc}-\mathrm{Mrp}$ or with $\mathrm{pBAD}$ TOPO was grown in LBS medium supplemented with $50 \mathrm{mM}$

\section{REFERENCES}

Aiyar, S. E., Gaal, T., and Gourse, R. L. (2002). rRNA promoter activity in the fast-growing bacterium Vibrio natriegens. J. Bacteriol. 184, 1349-1358. doi: 10.1128/JB.184.5.1349-1358.2002

Baba, T., Ara, T., Hasegawa, M., Takai, Y., Okumura, Y., Baba, M., et al. (2006). Construction of Escherichia coli K-12 in-frame, single-gene knockout mutants: the Keio collection. Mol. syst. biol. 2:2006.0008. doi: 10.1038/msb4100050
Tris $\mathrm{HCl} \mathrm{pH}$ 7.0, 0.1\% L-arabinose, $100 \mu \mathrm{g} / \mathrm{ml}$ ampicillin and varying amounts of $\mathrm{NaCl}, \mathrm{KCl}$, or $\mathrm{LiCl}$ as indicated. For end point measurements, $14 \mathrm{ml}$ sterile glass test tubes were used. Here, $10 \mathrm{ml}$ fresh medium was inoculated with $50 \mu \mathrm{l}$ cells from arabinose-induced culture (standardized to an $\mathrm{OD}_{600}=1$ ). The starting $\mathrm{OD}_{600}$ in all cases was 0.05 . After $20 \mathrm{~h}$ of incubation at $30{ }^{\circ} \mathrm{C}$ and moderate aeration, the optical density was measured at $600 \mathrm{~nm}$ (WPA biowave CO8000 cell density meter). To record growth curves, 96-well plates (Thermo Fisher Scientific) were used. A total of $200 \mu \mathrm{l}$ medium inoculated with induced cells was transferred into the plates and the growth was followed using a plate reader (Tecan infinite F200 Pro) measuring the absorbance at $600 \mathrm{~nm}$ every $13 \mathrm{~min}$ for $24 \mathrm{~h}$. The plate reader allowed shaking $(141 \mathrm{rpm})$ of the plate while incubation at a constant temperature of $37{ }^{\circ} \mathrm{C}$. To standard optical densities determined with the cell density meter and with the plate reader, we introduced a multiplication factor of 0.58 for the latter to account for the difference in optical path length (Toulouse et al., 2017).

\section{AUTHOR CONTRIBUTIONS}

LS designed the study, performed the experiments, analyzed the data, and drafted the manuscript. VM took part in designing the study. BC constructed the plasmid pNqrST. JP performed the mass spectrometry analyses. BB provided Vibrio natriegens and reviewed the manuscript. PD provided the plasmids pVc-Mrp and pBAD-TOPO and reviewed the manuscript. GF took part in designing the study and reviewed the manuscript. JS designed the study and wrote the manuscript.

\section{FUNDING}

This research was supported by grant FR1488/8-1 (to GF) and grant FR 1321/6-1 (to JS) from the Deutsche Forschungsgemeinschaft.

\section{SUPPLEMENTARY MATERIAL}

The Supplementary Material for this article can be found online at: https://www.frontiersin.org/articles/10.3389/fmicb. 2018.02537/full\#supplementary-material

Berger, I., Fitzgerald, D. J., and Richmond, T. J. (2004). Baculovirus expression system for heterologous multiprotein complexes. Nat. Biotechnol. 22, 1583-1587. doi: 10.1038/nbt1036

Bertsova, Y. V., Fadeeva, M. S., Kostyrko, V. A., Serebryakova, M. V., Baykov, A. A., and Bogachev, A. V. (2013). Alternative pyrimidine biosynthesis protein ApbE is a flavin transferase catalyzing covalent attachment of FMN to a threonine residue in bacterial flavoproteins. J. Biol. Chem. 288, 14276-14286. doi: 10.1074/ jbc.M113.455402 
Dzioba-Winogrodzki, J., Winogrodzki, O., Krulwich, T. A., Boin, M. A., Häse, C. C., and Dibrov, P. (2009). The Vibrio cholerae Mrp system: cation/proton antiport properties and enhancement of bile salt resistance in a heterologous host. J. Mol. Microbiol. Biotechnol. 16, 176-186. doi: 10.1159/000119547

Eagon, R. G. (1962). Pseudomonas natriegens, a marine bacterium with a generation time of less than 10 minutes. J. Bacteriol. 83, 736-737.

Failmezger, J., Scholz, S., Blombach, B., Siemann-Herzberg, M. (2018). Cell-free protein synthesis from fast-growing Vibrio natriegens. Front. Microbiol. 9:1146. doi: $10.3389 /$ fmicb. 2018.01146

Fitzgerald, D. J., Berger, P., Schaffitzel, C., Yamada, K., Richmond, T. J., and Berger, I. (2006). Protein complex expression by using multigene baculoviral vectors. Nat. Methods 3, 1021-1032. doi: 10.1038/nmeth983

Guzman, L. M., Belin, D., Carson, M. J., and Beckwith, J. (1995). Tight regulation, modulation, and high-level expression by vectors containing the arabinose PBAD promoter. J. Bacteriol. 177, 4121-4130. doi: 10.1128/jb.177.14.41214130.1995

Halang, P., Leptihn, S., Meier, T., Vorburger, T., and Steuber, J. (2013). The function of the $\mathrm{Na}+$-driven flagellum of Vibrio cholerae is determined by osmolality and pH. J. Bacteriol. 195, 4888-4899. doi: 10.1128/JB.00353-13

Hayashi, M., Nakayama, Y., and Unemoto, T. (2001). Recent progress in the $\mathrm{Na}+$-translocating NADH-quinone reductase from the marine Vibrio alginolyticus. Biochim. Biophys. Acta 1505, 37-44. doi: 10.1016/S0005-2728(00) 00275-9

Hoffart, E., Grenz, S., Lange, J., Nitschel, R., Müller, F., Schwentner, A., et al. (2017). High substrate uptake rates empower Vibrio natriegens as production host for industrial biotechnology. Appl. environ. microbiol. 83, e1614-e1617. doi: 10.1128/AEM.01614-17

Inoue, H., Nojima, H., and Okayama, H. (1990). High efficiency transformation of Escherichia coli with plasmids. Gene 96, 23-28. doi: 10.1016/0378-1119(90) 90336-P

Juárez, O., Athearn, K., Gillespie, P., and Barquera, B. (2009). Acid residues in the transmembrane helices of the $\mathrm{Na}+$-pumping $\mathrm{NADH}$ :quinone oxidoreductase from Vibrio cholerae involved in sodium translocation. Biochemistry 48, 9516-9524. doi: 10.1021/bi900845y

Kostyrko, V. A., Bertsova, Y. V., Serebryakova, M. V., Baykov, A. A., and Bogachev, A. V. (2015). NqrM (DUF539) protein is required for maturation of bacterial $\mathrm{Na}+$-translocating NADH:quinone oxidoreductase. J. Bacteriol. 198, 655-663. doi: 10.1128/JB.00757-15

Morino, M., Ogoda, S., Krulwich, T. A., and Ito, M. (2017). Differences in the phenotypic effects of mutations in homologous MrpA and MrpD subunits of the multi-subunit Mrp-type $\mathrm{Na}+/ \mathrm{H}+$ antiporter. Extremophiles 21, 51-64. doi: 10.1007/s00792-016-0877-z

Morino, M., Suzuki, T., Ito, M., and Krulwich, T. A. (2014). Purification and functional reconstitution of a seven-subunit mrp-type $\mathrm{Na}+/ \mathrm{H}+$ antiporter. J. Bacteriol. 196, 28-35. doi: 10.1128/JB.01029-13

Olsen, J. V., de Godoy, L. M., Li, G., Macek, B., Mortensen, P., Pesch, R., et al. (2005). Parts per million mass accuracy on an Orbitrap mass spectrometer via lock mass injection into a C-trap. Mol. Cell. proteomics 4, 2010-2021. doi: 10.1074/mcp.T500030-MCP200

Payne, W. J. (1958). Studies on bacterial utilization of uronic acids III. Induction of Oxidative Enzymes in a Marine Isolate1. J. bacteriol. 76, 301-307.

Pelosse, M., Crocker, H., Gorda, B., Lemaire, P., Rauch, J., and Berger, I. (2017). MultiBac: from protein complex structures to synthetic viral nanosystems. BMC Biol. 15:99. doi: 10.1186/s12915-017-0447-6

Rosano, G. L., and Ceccarelli, E. A. (2014). Recombinant protein expression in Escherichia coli: advances and challenges. Front. microbiol. 5:172. doi: 10.3389/ fmicb.2014.00172
Schägger, H., and von Jagow, G. (1987). Tricine-sodium dodecyl sulfatepolyacrylamide gel electrophoresis for the separation of proteins in the range from 1 to $100 \mathrm{kDa}$. Anal. Biochem. 166, 368-379. doi: 10.1016/0003-2697(87) 90587-2

Steuber, J., Halang, P., Vorburger, T., Steffen, W., Vohl, G., and Fritz, G. (2014a). Central role of the $\mathrm{Na}+$-translocating NADH:quinone oxidoreductase $(\mathrm{Na}+$ -NQR) in sodium bioenergetics of Vibrio cholerae. Biol. Chem. 395, 1389-1399. doi: 10.1515/hsz-2014-0204

Steuber, J., Vohl, G., Casutt, M. S., Vorburger, T., Diederichs, K., and Fritz, G. (2014b). Structure of the V. cholerae $\mathrm{Na}+$-pumping NADH:quinone oxidoreductase. Nature 516, 62-67. doi: 10.1038/nature14003

Steuber, J., Krebs, W., and Dimroth, P. (1997). The Na + -translocating NADH:ubiquinone oxidoreductase from vibrio alginolyticus. redox states of the FAD prosthetic group and mechanism of Ag + inhibition. Eur. J. Biochem. 249, 770-776. doi: 10.1111/j.1432-1033.1997.t01-2-00770.x

Swartz, T. H., Ikewada, S., Ishikawa, O., Ito, M., and Krulwich, T. A. (2005). The Mrp system: a giant among monovalent cation/proton antiporters? Extremophiles 9, 345-354. doi: 10.1007/s00792-005-0451-6

Tao, M., Casutt, M. S., Fritz, G., and Steuber, J. (2008). Oxidant-induced formation of a neutral flavosemiquinone in the $\mathrm{Na}+$-translocating NADH:Quinone oxidoreductase (Na + -NQR) from Vibrio cholerae. Biochim. Biophys. Acta 1777, 696-702. doi: 10.1016/j.bbabio.2008.04.006

Tolia, N. H., and Joshua-Tor, L. (2006). Strategies for protein coexpression in Escherichia coli. Nat. Methods 3, 55-64. doi: 10.1038/nmeth0106-55

Toulouse, C., Häse, C. C., and Steuber, J. (2017). Chloroform-free permeabilization for improved detection of $\beta$-galactosidase activity in Vibrio cholerae. J. Microbiol. Methods 137, 1-2. doi: 10.1016/j.mimet.2017.03.011

Vohl, G., Nedielkov, R., Claussen, B., Casutt, M. S., Vorburger, T., Diederichs, K., et al. (2014). Crystallization and preliminary analysis of the NqrA and NqrC subunits of the $\mathrm{Na}+$-translocating NADH:ubiquinone oxidoreductase from Vibrio cholerae. Acta crystallogr. F Struct. Biol. Commun. 70, 987-992. doi: 10.1107/S2053230X14009881

Vorburger, T., Nedielkov, R., Brosig, A., Bok, E., Schunke, E., Steffen, W., et al. (2016). Role of the $\mathrm{Na}+$-translocating NADH:quinone oxidoreductase in voltage generation and $\mathrm{Na}+$ extrusion in Vibrio cholerae. Biochim. Biophys. Acta 1857, 473-482. doi: 10.1016/j.bbabio.2015.12.010

Weinstock, M. T., Hesek, E. D., Wilson, C. M., and Gibson, D. G. (2016). Vibrio natriegens as a fast-growing host for molecular biology. Nat. Methods 13, 849-851. doi: 10.1038/nmeth.3970

Yan, L. J., Yang, S. H., Shu, H., Prokai, L., and Forster, M. J. (2007). Histochemical staining and quantification of dihydrolipoamide dehydrogenase diaphorase activity using blue native PAGE. Electrophoresis 28, 1036-1045.

Zorman, S., Botte, M., Jiang, Q., Collinson, I., and Schaffitzel, C. (2015). Advances and challenges of membrane-protein complex production. Curr. Opin. Struct. Biol. 32, 123-130. doi: 10.1016/j.sbi.2015.03.010

Conflict of Interest Statement: The authors declare that the research was conducted in the absence of any commercial or financial relationships that could be construed as a potential conflict of interest.

Copyright () 2018 Schleicher, Muras, Claussen, Pfannstiel, Blombach, Dibrov, Fritz and Steuber. This is an open-access article distributed under the terms of the Creative Commons Attribution License (CC BY). The use, distribution or reproduction in other forums is permitted, provided the original author(s) and the copyright owner(s) are credited and that the original publication in this journal is cited, in accordance with accepted academic practice. No use, distribution or reproduction is permitted which does not comply with these terms. 